\title{
Determination of Macro and Trace Element Levels in Honey from the Lower Amazonian Region, Brazil
}

\author{
Gabriela Sousa Dourado1, Victor Valentin Gomes ${ }^{1}$, Maila Thais Vieira Maia1, Arthur Abinader \\ Vasconcelos $^{1}$, Kauê Santana da Costa ${ }^{1}$, Kelson do Carmo Faial ${ }^{2}$, Bruno Santana Carneiro ${ }^{2}$, \\ Newton Trindade Vasconcelos Junior ${ }^{2}$, Paulo Sérgio Taube ${ }^{1} \bigotimes$ iD \\ ${ }^{1}$ Instituto de Biodiversidade e Florestas, Universidade Federal do Oeste do Pará, Rua Vera Paz s/n, \\ 68005-100, Santarém, PA, Brazil \\ ${ }^{2}$ Instituto Evandro Chagas, Campus de Ananindeua, Rod. BR-316 Km 7 S/N, 67000-030, Ananindeua, PA, Brazil
}

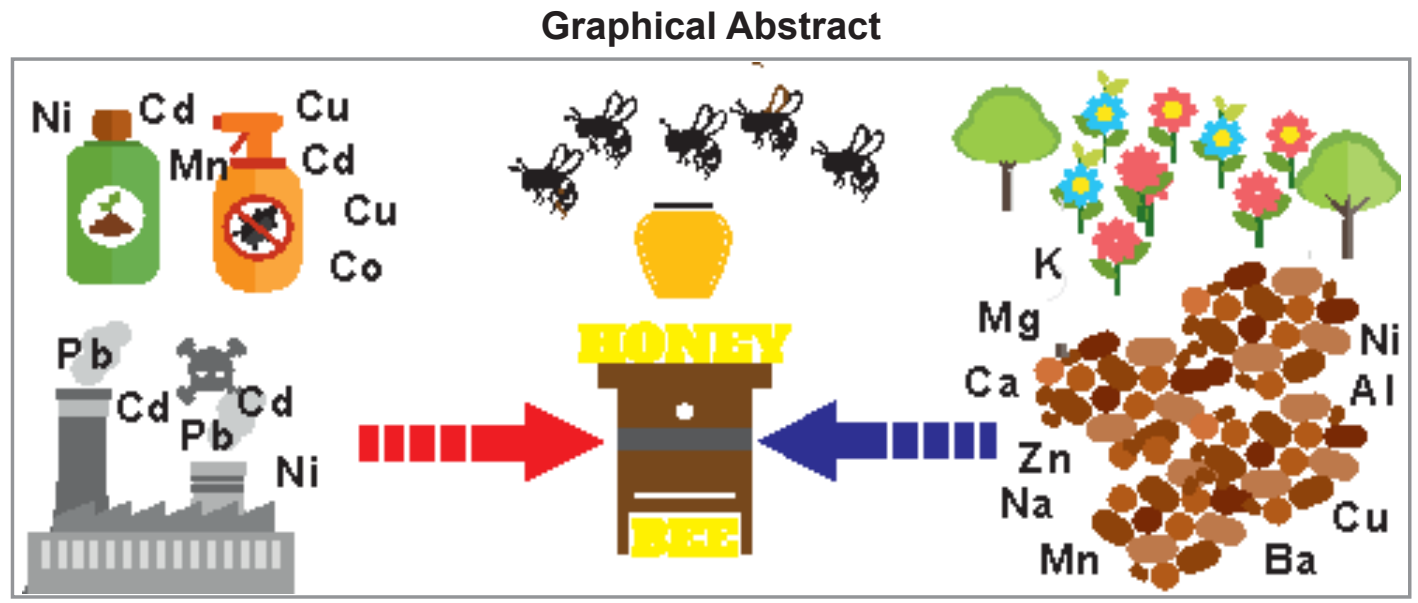

Possible sources of macro and micronutients, as well as potentially toxic elements in honey

The aim of this study was to quantify the macro and trace element concentrations in Apis mellifera and Melipona interrupta honey samples from the Lower Amazonian region in order to evaluate if the samples of different geographical origin and/or species could be distinguished by their mineral content. In addition, it was evaluated the presence of potentially toxic metals in honey samples. The metal contents were determined by inductively coupled plasma optical emission spectrometry (ICP OES) and the most abundant metals found in the samples were $\mathrm{K}, \mathrm{Ca}, \mathrm{Mg}$, and $\mathrm{Na}$. The total metal $(\mathrm{K}, \mathrm{Ca}, \mathrm{Mg}$, $\mathrm{Na}, \mathrm{Cd}, \mathrm{Co}, \mathrm{Ni}, \mathrm{Fe}, \mathrm{Mn}, \mathrm{Cr}, \mathrm{Al}$ and $\mathrm{Ba}$ ) concentration ranged from $127.7 \pm 1.4$ to $1844.4 \pm 45.2 \mu \mathrm{g} \mathrm{g}^{-1}$ and from $102.7 \pm 2.0$ to $639.0 \pm 15.3 \mu \mathrm{g} \mathrm{g}^{-1}$ in Apis mellifera and Melipona interrupta honey, respectively. All mineral content levels found in the honey samples were lower than the maximum established by Brazilian and international law (Cd and $\mathrm{Cr} 0.1 \mu \mathrm{g} \mathrm{g}^{-1}$, Pb $0.30 \mu \mathrm{g} \mathrm{g}^{-1}$, Ni $5 \mu \mathrm{g} \mathrm{g}^{-1}, \mathrm{Cu} 10 \mu \mathrm{g} \mathrm{g}{ }^{-1}, \mathrm{Zn}_{50}$ $\mu \mathrm{g} \mathrm{g}^{-1}$ ). Furthermore, $\mathrm{Cu}, \mathrm{Pb}$, and $\mathrm{Zn}$ were not detected in any of the samples. However, potentially toxic elements, such as $\mathrm{Cd}$, Co, and $\mathrm{Ni}$, were detected in most of the commercial samples and in Apis mellifera honey from beehives that were close to livestock fields and/or soybean areas. Hierarchical cluster analysis (HCA) was used to study the mineral contents and it was possible to distinguish eight different groups of honey. However, the Melipona interrupta honey could not be separated into different groups.

Keywords: Trace elements, mineral content, toxic metals, hierarchical component analysis 


\section{INTRODUCTION}

The mineral composition of honey consists of approximately 27 elements, and several studies have reported that it is possible to separate honey from different floral and geographical origins using the presence of specific mineral groups [1-3]. These metals are classified into macro elements, such as: $\mathrm{K}, \mathrm{P}$, and $\mathrm{Ca}$, and trace elements, such as: $\mathrm{Pb}, \mathrm{Cd}, \mathrm{Zn}, \mathrm{Fe}, \mathrm{Mg}, \mathrm{Mn}, \mathrm{Al}, \mathrm{Ba}, \mathrm{Ag}, \mathrm{Cr}$, As, etc., which are not considered to be essential components of honey [4]. The trace element content in honey varies, but it is typically below $1.0 \mu \mathrm{g} \mathrm{g}^{-1}$ and is normally associated with its color. For example, dark honey has the highest metal contents [5-6]. Brazil is one of the largest producers of honey (FAO 2014) [7] and its production is widely distributed across all regions of the country, which has led to a large variety of honey types [8]. The Lower Amazonian region is a promising area for beekeeping due to its special flora and climate characteristics, although it is considered to be a secondary activity [8]. However, this region has been suffering from agricultural expansion since the 1970s, and has mainly occurred along the BR 163 highway [9].

Several potentially toxic elements bioaccumulate in soils and could affect plant and, consequently, honey quality. Therefore, it is important to evaluate the mineral composition of honey, especially because some minerals are essential for human health. It is also important because some trace elements, such as $\mathrm{Cd}, \mathrm{Hg}$, and $\mathrm{Pb}$, are non-essential but toxic in very small concentrations and some, such as $\mathrm{Cr}$, Fe, $\mathrm{Mn}, \mathrm{Ni}, \mathrm{Se}$, and $\mathrm{Zn}$ are essential at certain content levels, but can bioaccumulate to toxic levels (plantanimal-human) under certain circumstances [5].

It has been suggested that honey could be used as bio-indicator of environmental pollution if it is produced from the nectar of different plants, particularly if the nectar bioaccumulates heavy metals [5]. However, honey could also be contaminated with different minerals during processing and storage (e.g., contaminated equipment and tools, and poor hygiene) [6,10-11].

Some authors have found large concentrations of heavy metals in honey produced in areas where industrial and/or agricultural activity is high. These types of activity are considered the most important sources of honey contamination [12-13]. The use of multivariate analysis methods such as principal component analysis (PCA) and hierarchical cluster analysis (HCA) have been used for statistical data processing in order to classify honey samples according to their properties in different groups according to their botanical origin, species or geographical origin [4,6,14-18].

The first aim of this study was to determine the metal contents (macro and trace elements) in Apis mellifera and Melipona interrupta honeys from the Lower Amazonian region. Secondly, to evaluate whether it is possible to differentiate honey by its geographical origin or bee species through the determination of its mineral content. In addition, the degree of contamination of these samples by potentially toxic metals was evaluated.

\section{MATERIALS AND METHODS Honey samples}

A total of 46 honey samples from the Lower Amazonian region were collected between November 2015 and July 2017. Sixteen samples were obtained from commercial sources and 30 samples were obtained through manual extraction where no metal instruments were used and without the use of smoke or chemicals. These samples were collected from two bee species. A total of 33 samples came from Apis mellifera (M01-M33) and 13 samples came from Jandaíra (Melipona interrupta) (J01-J13). Thirty five samples (M01-M20, M30-M33, and J1-J11), four samples (M26-M29), and seven samples (M21M25 and J12-J13) were obtained from Santarém (02 $26^{\prime} 34^{\prime \prime} \mathrm{S}$ and $\left.54^{\circ} 42^{\prime 2} 28^{\prime \prime} \mathrm{W}\right)$, Belterra $\left(02^{\circ} 38^{\prime} 09^{\prime \prime S}\right.$

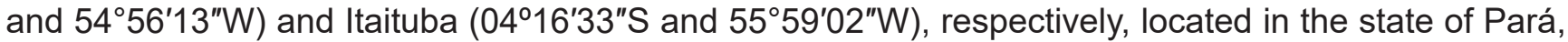
Brazil (Figure 1 and Table I). All honey samples were stored in Falcon tubes, protected from light, and kept at room temperature until needed for further analysis. 

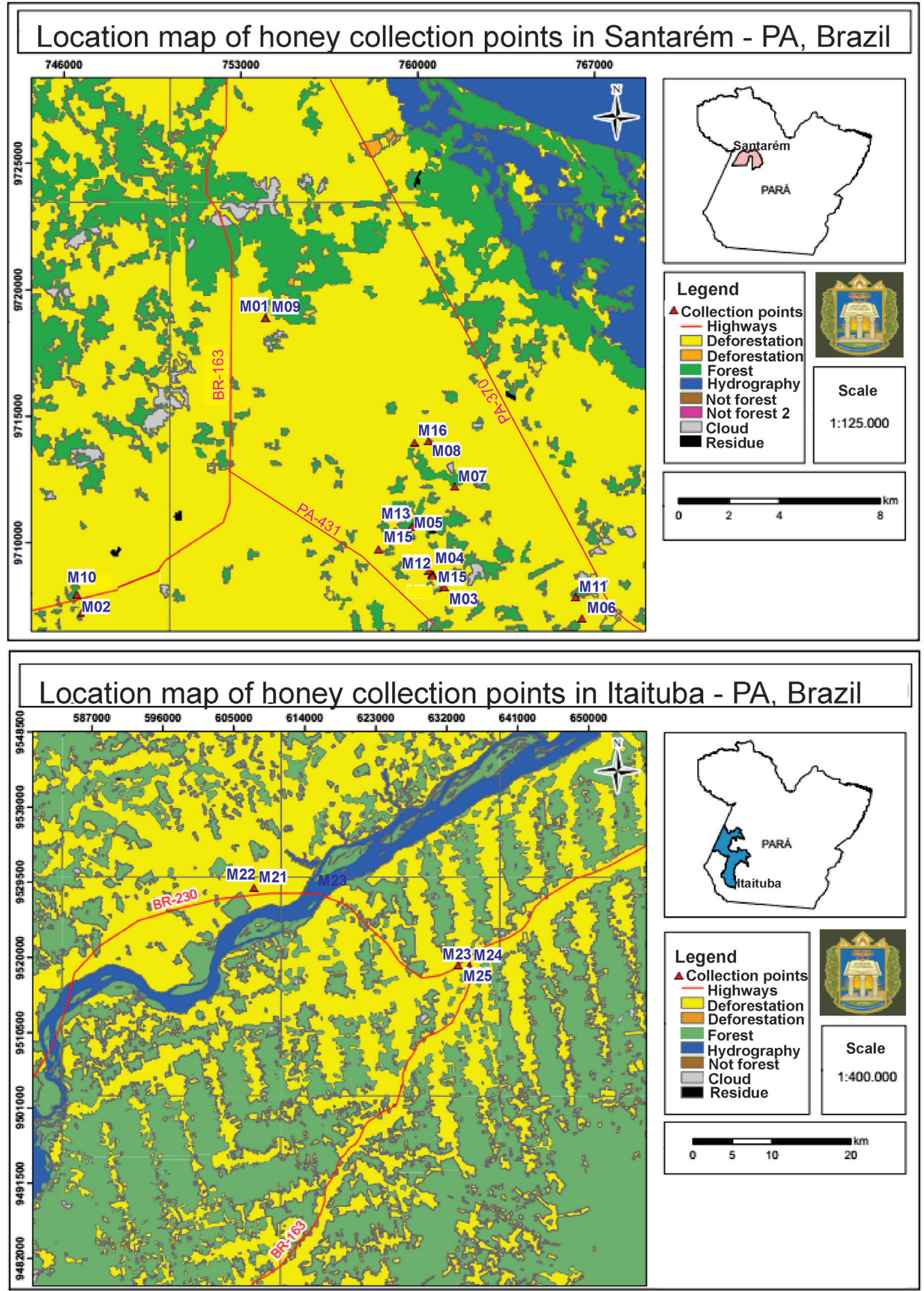

Figure 1. Geographic location of the beehives where the honey samples were collected. 
Table I. Main characteristics of the different beehive areas

\begin{tabular}{|c|c|}
\hline ID sample & Description of the beehive areas \\
\hline M01 & Soybean field $\pm 300 \mathrm{~m}$, highway $\pm 500 \mathrm{~m}$, obtained from the beestock \\
\hline M02 & $\begin{array}{l}\text { Located in a secondary forest, Livestock area } \pm 500 \text {, highway } \pm 300 \mathrm{~m} \text {, obtained from the } \\
\text { beestock }\end{array}$ \\
\hline M03 & Soybean field and livestock area $\pm 500 \mathrm{~m}$, highway $\pm 2000 \mathrm{~m}$, obtained from the beestock \\
\hline M04 & $\begin{array}{l}\text { Located in secondary forest, passion fruit plantation } \pm 300 \mathrm{~m} \text {, highway } \pm 1500 \mathrm{~m} \text {, obtained } \\
\text { from the beestock }\end{array}$ \\
\hline M05 & Located in secondary forest, highway $\pm 2000 \mathrm{~m}$, obtained from the beestock \\
\hline M06 & Soybean field $\pm 500 \mathrm{~m}$, highway $\pm 1000 \mathrm{~m}$, obtained from the beestock \\
\hline M07 & Livestock area $\pm 500 \mathrm{~m}$, highway $\pm 3000 \mathrm{~m}$, obtained from the beestock \\
\hline M08 & $\begin{array}{l}\text { Vegetable garden } \pm 1000 \mathrm{~m} \text {, livestock area } \pm 1500 \mathrm{~m} \text {, highway } \pm 3000 \mathrm{~m} \text {, obtained from the } \\
\text { beestock }\end{array}$ \\
\hline M09 & Soybean field $\pm 500 \mathrm{~m}$, highway $\pm 1000 \mathrm{~m}$, obtained from the beestock \\
\hline M10 & $\begin{array}{l}\text { Located in secondary forest, livestock area } \pm 500 \mathrm{~m} \text {, highway } \pm 300 \mathrm{~m} \text {, obtained from the } \\
\text { beestock }\end{array}$ \\
\hline M11 & Soybean field and livestock area $\pm 500 \mathrm{~m}$, highway $\pm 2000 \mathrm{~m}$, obtained from the beestock \\
\hline M12 & $\begin{array}{l}\text { Located in secondary forest, passion fruit plantation } \pm 500 \mathrm{~m} \text {, highway } \pm 1500 \mathrm{~m} \text {, obtained } \\
\text { from the beestock }\end{array}$ \\
\hline M13 & Located in secondary forest, highway $\pm 2000 \mathrm{~m}$, obtained from the beestock \\
\hline M14 & Soybean field $\pm 1000 \mathrm{~m}$, highway $\pm 1500 \mathrm{~m}$, obtained from the beestock \\
\hline M15 & Livestock area $\pm 500 \mathrm{~m}$, highway $\pm 300 \mathrm{~m}$, obtained from the beestock \\
\hline M16 & $\begin{array}{l}\text { Vegetable garden } \pm 1000 \mathrm{~m} \text {, livestock area } \pm 1500 \mathrm{~m} \text {, highway } \pm 3000 \mathrm{~m} \text {, obtained from the } \\
\text { beestock }\end{array}$ \\
\hline M17 & Commercial sample \\
\hline M18 & Commercial sample \\
\hline M19 & Commercial sample \\
\hline M20 & Commercial sample \\
\hline M21 & Located in secondary forest, freshly harvested honey \\
\hline M22 & Located in secondary forest, freshly harvested honey \\
\hline M23 & $\begin{array}{l}\text { Acerola (Malpighia emarginata), cupuaçu (Theobroma grandiflorum), papay (Carica pa- } \\
\text { paya), orange (Citrus aurantium), and lemon (Citrus limonum) trees. }\end{array}$ \\
\hline M24 & $\begin{array}{l}\text { Acerola (Malpighia emarginata), cupuaçu (Theobroma grandiflorum), papay (Carica pa- } \\
\text { paya), orange (Citrus aurantium), and lemon (Citrus limonum) trees. }\end{array}$ \\
\hline M25 & $\begin{array}{l}\text { Acerola (Malpighia emarginata), cupuaçu (Theobroma grandiflorum), papay (Carica pa- } \\
\text { paya), orange (Citrus aurantium), and lemon (Citrus limonum) trees. }\end{array}$ \\
\hline M26 & $\begin{array}{l}\text { Mango (Mangifera indica L.), orange (Citrus aurantium), Brazil nut (Bertholletia excelsa), } \\
\text { cashew (Anacardium occidentale) and açai (Euterpe oleracea) trees. }\end{array}$ \\
\hline M27 & $\begin{array}{l}\text { Mango (Mangifera indica L.), orange (Citrus aurantium), Brazil nut (Bertholletia excelsa), } \\
\text { cashew (Anacardium occidentale), and açai (Euterpe oleracea) trees. }\end{array}$ \\
\hline M28 & $\begin{array}{l}\text { Mango (Mangifera indica L.), orange (Citrus aurantium), Brazil nut (Bertholletia excelsa), } \\
\text { cashew (Anacardium occidentale), and açai (Euterpe oleracea) trees. }\end{array}$ \\
\hline M29 & $\begin{array}{l}\text { Mango (Mangifera indica L.), orange (Citrus aurantium), Brazil nut (Bertholletia excelsa), } \\
\text { cashew (Anacardium occidentale), and açai (Euterpe oleracea) trees. }\end{array}$ \\
\hline M30 & Commercial sample \\
\hline M31 & Commercial sample \\
\hline M32 & Commercial sample \\
\hline M33 & Commercial sample \\
\hline
\end{tabular}


Table I. Main characteristics of the different beehive areas (Cont)

\begin{tabular}{ll}
\hline ID sample & Description of the beehive areas \\
\hline $\mathrm{J} 01$ & Located in secondary forest, soybean field $\pm 1000 \mathrm{~m}$, highway $\pm 1000 \mathrm{~m}$ \\
$\mathrm{~J} 02$ & Located in secondary forest, soybean field $\pm 1000 \mathrm{~m}$, highway $\pm 1000 \mathrm{~m}$ \\
$\mathrm{~J} 03$ & Located in secondary forest, soybean field $\pm 1000 \mathrm{~m}$, passion fruit plantation $\pm 300 \mathrm{~m}$, high- \\
& way $\pm 1500 \mathrm{~m}$ \\
$\mathrm{~J} 04$ & Soybean field $\pm 1000 \mathrm{~m}$, highway $\pm 1000 \mathrm{~m}$ \\
$\mathrm{~J} 05$ & Located in a secondary forest, soybean field $\pm 1000 \mathrm{~m}$, highway $\pm 1000 \mathrm{~m}$ \\
$\mathrm{~J} 06$ & Commercial sample \\
$\mathrm{J} 07$ & Commercial sample \\
$\mathrm{J} 08$ & Commercial sample \\
$\mathrm{J} 09$ & Commercial sample \\
$\mathrm{J} 10$ & Commercial sample \\
$\mathrm{J} 11$ & Commercial sample \\
$\mathrm{J} 12$ & Commercial sample \\
$\mathrm{J} 13$ & Commercial sample \\
\hline
\end{tabular}

M: Apis mellifera honey; J: Jandaíra (Melipona interrupta) honey.

\section{Sample preparation}

\section{Microwave assisted digestion}

Approximately $1.0 \mathrm{~g}$ of the sample was weighed in polytetrafluoroethylene (PTFE) tubes and then mixed with $2.0 \mathrm{~mL}$ of $\mathrm{HNO}_{3}, 14.0$ mol L-1 (Vetec, Rio de Janeiro, RJ, Brazil) that had been previously distillated in a quartz distillatory (Kurner, Analysentechnik, Rosenheim, Germany); $2.0 \mathrm{~mL}$ of $\mathrm{H}_{2} \mathrm{O}_{2}$, $30 \%(\mathrm{v} / \mathrm{v})$ (Vetec, Rio de Janeiro, RJ, Brazil); and $0.5 \mathrm{~mL}$ of yttrium solution $\left(100.0 \mathrm{mg} \mathrm{L}^{-1}\right)$ (Sigma Aldrich, St. Louis, MO, USA), which was the internal standard. The mixture was divided into three fractions and then was subjected to a heating program in a closed microwave oven (CEM/Mars Xpress, Matthews, NC, USA), which consisted of the following steps: 1 min at $320 \mathrm{~W}, 2$ min at $120 \mathrm{~W}, 5 \mathrm{~min}$ at $320 \mathrm{~W}, 5 \mathrm{~min}$ at $520 \mathrm{~W}$, and $5 \mathrm{~min}$ at $720 \mathrm{~W}$. Then, the resulting mixture was left to cool and was quantitatively transferred to polypropylene vials. The volume of the final solution was made up to 25.0 $\mathrm{mL}$ with ultrapure water with a resistivity of $18 \mathrm{M} \Omega \mathrm{cm}$. The ultrapure water had been obtained from a Milli-Q system (Millipore, Bedford, MA, USA) before analysis. Reagent blank containing ultrapure water, $\mathrm{HNO}_{3}$, and $\mathrm{H}_{2} \mathrm{O}_{2}$ was subjected to the same treatment as the honey samples.

The metal contents in the honey were determined by inductively coupled plasma optical emission spectroscopy (ICP OES) model Vista-MPX CCD (Varian, Mulgrave, VIC, Australia) with an automatic sampling system (ASX 520, Cetac Technologies, Omaha, NE, USA). Each sample was analyzed in triplicate. A cyclonic spray chamber and concentric nebulizer were used. The metal determinations were carried out using the manufacturer recommended conditions for power $(1.4 \mathrm{~kW})$, generator frequency (40 MHz), plasma gas flow (15 L min-1), auxiliary gas flow $\left(1.5 \mathrm{~L} \mathrm{~min}^{-1}\right)$, nebulizer gas flow $\left(0.75 \mathrm{~L} \mathrm{~min}^{-1}\right)$ and sample uptake rate $\left(1.0 \mathrm{~mL} \mathrm{~min}^{-1}\right)$. The emission intensity scan duration was $60 \mathrm{~s}$. The following analytical wavelengths (nm) were selected: Al (396.152), Ba (455.403), Ca (318.127), Cd (226.502), Co (231.160) Cr (276.716), Cu (327.395), Fe (238.204), K (766.491), Mg (280.270), Mn (257.610), Na (589.592), Ni (231.604), Pb (220.353) and Zn (213.857).

The absence of certified honey samples meant that recovery tests were performed to validate the method. The recovery percentage was calculated by processing five honey samples spiked with known amounts of element analytical standards.

Calibration of the samples against matrix matches was undertaken so that the macro and trace element concentrations could be calculated and to correct for matrix interferences. One sample of base 
honey from Apis mellifera (M10) honey was selected. Then, standard stock solutions containing 1000 $\mathrm{mg} \mathrm{L}^{-1}$ of $\mathrm{Zn}, \mathrm{Pb}, \mathrm{Ni}, \mathrm{Cd}$, Co, $\mathrm{Fe}, \mathrm{Mn}, \mathrm{Cr}, \mathrm{Mg}, \mathrm{Cu}, \mathrm{Al}, \mathrm{Ca}, \mathrm{Ba}, \mathrm{Na}$, or $\mathrm{K}$ were purchased from Merck (Darmstadt, Germany). Analytical calibration standards were prepared daily and the concentrations ranged from 40 to $400 \mathrm{\mu g} \mathrm{L}^{-1}$ for all analyzed elements. The base honey was prepared in the same manner as the samples. Yttrium was added as the internal standard to a final concentration $10 \mu \mathrm{g} \mathrm{L}^{-1}$. This sample was digested in a microwave oven and after dilution in the same way as the other samples.

The limits of detection (LOD) were calculated using the equation LOD $=(3 \times$ RSDBlank $\times$ BEC $) / 100$ and $L O Q=(10 \times$ RSDBlank $\times B E C) / 100$. The limits of quantification (LOQ) were determined as three times the LOD value ( $3 \times$ LOD). Where BEC (background equivalent concentration) is the blank intensity divided by the angular coefficient of the analytical curve and RSDBlank is the relative standard deviation of the blank $(n=10)$. The equation for the BEC can also be written as BEC $=C_{\text {Element }} / S B R$, where $\mathrm{C}_{\text {Element }}$ is the element concentration and SBR (signal-bottom ratio) is the ratio between the intensity of the pure analytical signal and the background signal, which can be calculated SBR $=\left(I_{\text {Analytical }}-I_{\text {Blank }}\right) /$ $\mathrm{I}_{\text {Blank. }}$. The LOD and LOQ values are given in Table II.

All analyses were run in triplicate and the results were expressed as means \pm the standard deviation (SD). A Pearson's correlation analysis was performed on the mean values for each metal content using Minitab Statistical Software 14 (Minitab, State College, PA, USA).

Statistical multivariate analysis by Principal Component Analysis (PCA) and Hierarchical Cluster Analysis (HCA) were undertaken using the complete linkage with the square Euclidian distance to evaluate if Apis mellifera and Melipona interrupta honey could be distinguished by their mineral contents. The results of the analysis are presented in a dendrogram plot that shows the similarity level between sample results provided by the Minitab Statistical Software 14 (Minitab Inc., 2010) and free software R version 3.1.1 (R Core Team, 2017).

\section{RESULTS AND DISCUSSION}

\section{Macro and trace element concentrations in honey from different areas of Brazil}

Table II shows the limits of detection and quantification as well as the percentage of recovery of the analyzed elements. The recovery obtained for all elements ranged from $67.0 \%(\mathrm{Al})$ to $113.1 \%(\mathrm{~K})$.

A total of 15 metals were simultaneously determined using ICP OES and the average macro and trace element concentrations in the honey samples are given along with their standard deviations in Tables IV and V, respectively.

Total metal concentration was obtained from the sum of the mean concentrations of each element, which ranged from 127.7 (M26) to $1844.4 \mu \mathrm{g} \mathrm{g}^{-1}$ (M23) and from 102.7 (J05) to $639.0 \mu \mathrm{g} \mathrm{g}^{-1}$ (J07) for Apis mellifera and Melipona interrupta, respectively. The most abundant elements found in the honey samples were $\mathrm{K}, \mathrm{Na}, \mathrm{Ca}$, and $\mathrm{Mg}$ with maximum concentrations of $1618.0 \mu \mathrm{g} \mathrm{g}^{-1}$ (M23), $200.3 \mu \mathrm{g} \mathrm{g}{ }^{-1}$ (M32), $167.5 \mu^{-1} g^{-1}$ (M32), and $134.2 \mu g^{-1}$ (M32), respectively (Table III).

Table II. Major (Mg, Ca, Na, K) and trace element (Zn, Pb, Cd, Co, Ni, Fe, Mn, Cr, Cu, Al, Ba) levels, and recoveries from spiked honey samples

\begin{tabular}{cccccc}
\hline Element & LOD $\left(\mathbf{n g ~ ~ ^ { - 1 } )}\right.$ & LOQ $\left(\mathbf{n g ~ ~ ^ { - 1 } )}\right.$ & $\begin{array}{c}\text { Spike values } \\
\left(\boldsymbol{\mu \mathbf { g ~ g ~ } ^ { - 1 } )}\right.\end{array}$ & $\begin{array}{c}\text { Measured } \\
\text { value }\left(\boldsymbol{\mu \mathbf { g ~ g } ^ { - 1 } )}\right.\end{array}$ & Recovery (\%) \\
\hline $\mathrm{Mg}$ & 0.19 & 0.66 & 500 & $391.67 \pm 12.89$ & $76.6 \pm 2.2$ \\
$\mathrm{Ca}$ & 0.36 & 1.17 & 500 & $425.35 \pm 15.39$ & $81.6 \pm 3.3$ \\
$\mathrm{Na}$ & 0.29 & 0.96 & 500 & $486.54 \pm 39.92$ & $95.5 \pm 6.6$ \\
$\mathrm{~K}$ & 1.59 & 5.30 & 500 & $565.50 \pm 34.90$ & $113.1 \pm 7.0$ \\
$\mathrm{Zn}$ & 0.23 & 0.77 & 500 & $410.66 \pm 16.32$ & $82.1 \pm 3.3$ \\
$\mathrm{~Pb}$ & 0.21 & 0.69 & 500 & $448.44 \pm 17.20$ & $89.7 \pm 3.4$
\end{tabular}


Table II. Major (Mg, Ca, Na, K) and trace element (Zn, Pb, Cd, Co, Ni, Fe, Mn, Cr, Cu, Al, Ba) levels, and recoveries from spiked honey samples (Cont)

\begin{tabular}{cccccc}
\hline Element & LOD $\left(\mathbf{n g ~ \mathbf { ~ } ^ { - 1 } )}\right.$ & LOQ $\left(\mathbf{n g ~ \mathbf { ~ } ^ { - 1 } )}\right.$ & $\begin{array}{c}\text { Spike values } \\
\left(\boldsymbol{\mu \mathbf { g ~ g } ^ { - 1 } )}\right.\end{array}$ & $\begin{array}{c}\text { Measured } \\
\text { value }\left(\boldsymbol{\mu g} \mathbf{~ g}^{-1}\right)\end{array}$ & Recovery (\%) \\
\hline $\mathrm{Cd}$ & 1.30 & 4.34 & 500 & $374.67 \pm 16.39$ & $74.9 \pm 3.3$ \\
$\mathrm{Co}$ & 0.87 & 2.90 & 500 & $372.05 \pm 17.50$ & $74.4 \pm 3.5$ \\
$\mathrm{Ni}$ & 2.71 & 9.04 & 500 & $372.79 \pm 13.98$ & $74.6 \pm 2.8$ \\
$\mathrm{Fe}$ & 0.28 & 0.93 & 500 & $378.70 \pm 17.24$ & $75.7 \pm 3.4$ \\
$\mathrm{Mn}$ & 0.40 & 1.34 & 500 & $374.67 \pm 16.39$ & $74.9 \pm 3.3$ \\
$\mathrm{Cr}$ & 1.66 & 5.53 & 500 & $391.40 \pm 16.34$ & $78.3 \pm 3.3$ \\
$\mathrm{Cu}$ & 1.19 & 3.99 & 500 & $385.11 \pm 15.70$ & $77.0 \pm 3.1$ \\
$\mathrm{Al}$ & 0.28 & 0.94 & 500 & $335.23 \pm 13.84$ & $67.0 \pm 2.8$ \\
$\mathrm{Ba}$ & 1.13 & 3.79 & 500 & $337.29 \pm 12.11$ & $67.5 \pm 2.4$ \\
\hline
\end{tabular}

LOD: Limits of detection LOQ: Limits of quantification

Table III. Average Mg, Ca, $\mathrm{Na}$, and $\mathrm{K}$ concentrations in the honey samples $\left.\left(\mu_{\mathrm{g} \mathrm{g}}\right)^{-1}\right)^{\mathrm{a}}$

\begin{tabular}{|c|c|c|c|c|}
\hline ID sample & Mg & $\mathrm{Ca}$ & $\mathrm{Na}$ & $\mathbf{K}$ \\
\hline M01 & $21.98 \pm 0.44$ & $43.39 \pm 0.35$ & $42.18 \pm 0.49$ & $364.87 \pm 7.13$ \\
\hline M02 & $8.82 \pm 0.20$ & $22.32 \pm 0.17$ & $28.04 \pm 0.17$ & $201.71 \pm 5.25$ \\
\hline M03 & $19.55 \pm 0.23$ & $41.91 \pm 0.56$ & $51.50 \pm 0.50$ & $448.17 \pm 10.79$ \\
\hline M04 & $27.18 \pm 0.30$ & $47.67 \pm 0.72$ & $73.40 \pm 0.58$ & $592.40 \pm 15.36$ \\
\hline M05 & $60.06 \pm 0.19$ & $117.63 \pm 0.52$ & $82.91 \pm 0.86$ & $1418.06 \pm 30.12$ \\
\hline M06 & $51.39 \pm 0.20$ & $89.22 \pm 0.91$ & $90.12 \pm 1.00$ & $802.92 \pm 6.69$ \\
\hline M07 & $71.14 \pm 0.20$ & $131.02 \pm 0.49$ & $103.91 \pm 1.10$ & $1404.06 \pm 42.84$ \\
\hline M08 & $88.68 \pm 0.43$ & $113.76 \pm 0.52$ & $98.40 \pm 0.44$ & $1465.84 \pm 27.13$ \\
\hline M09 & $27.85 \pm 0.46$ & $51.72 \pm 0.41$ & $69.31 \pm 0.57$ & $483.35 \pm 4.86$ \\
\hline M10 & $12.05 \pm 0.27$ & $26.78 \pm 0.32$ & $68.21 \pm 1.82$ & $234.47 \pm 6.41$ \\
\hline M11 & $20.24 \pm 0.36$ & $42.91 \pm 0.49$ & $81.62 \pm 1.34$ & $441.82 \pm 8.76$ \\
\hline M12 & $13.74 \pm 0.28$ & $23.07 \pm 1.07$ & $55.59 \pm 0.78$ & $233.42 \pm 13.32$ \\
\hline M13 & $36.37 \pm 0.19$ & $70.25 \pm 0.66$ & $83.02 \pm 1.07$ & $761.18 \pm 3.04$ \\
\hline M14 & $36.73 \pm 0.26$ & $59.46 \pm 1.24$ & $89.99 \pm 0.96$ & $568.89 \pm 5.92$ \\
\hline M15 & $43.66 \pm 0.50$ & $81.96 \pm 0.35$ & $87.80 \pm 0.64$ & $695.98 \pm 20.12$ \\
\hline M16 & $45.46 \pm 0.41$ & $63.25 \pm 0.89$ & $82.94 \pm 0.28$ & $737.39 \pm 12.80$ \\
\hline M17 & $13.86 \pm 0.55$ & $98.41 \pm 1.90$ & $137.33 \pm 3.43$ & $667.00 \pm 2.17$ \\
\hline M18 & $7.99 \pm 0.36$ & $17.11 \pm 0.44$ & $67.19 \pm 2.57$ & $161.83 \pm 2.13$ \\
\hline M19 & $15.28 \pm 0.75$ & $29.92 \pm 1.23$ & $68.26 \pm 1.64$ & $333.25 \pm 6.18$ \\
\hline M20 & $23.83 \pm 0.38$ & $29.21 \pm 2.79$ & $62.34 \pm 1.12$ & $198.45 \pm 2.28$ \\
\hline M21 & $9.26 \pm 0.21$ & $36.74 \pm 0.46$ & $59.98 \pm 0.66$ & $118.86 \pm 6.75$ \\
\hline
\end{tabular}


Table III. Average Mg, $\mathrm{Ca}, \mathrm{Na}$, and $\mathrm{K}$ concentrations in the honey samples $\left(\mu \mathrm{g} \mathrm{g}^{-1}\right)^{\mathrm{a}}$ (Cont)

\begin{tabular}{|c|c|c|c|c|}
\hline ID sample & Mg & $\mathrm{Ca}$ & $\mathrm{Na}$ & $\mathbf{K}$ \\
\hline M22 & $9.32 \pm 0.11$ & $42.63 \pm 0.44$ & $72.02 \pm 0.53$ & $68.69 \pm 4.59$ \\
\hline M23 & $34.91 \pm 0.13$ & $103.17 \pm 0.62$ & $81.28 \pm 0.52$ & $1618.01 \pm 43.76$ \\
\hline M24 & $35.62 \pm 0.10$ & $74.44 \pm 0.54$ & $60.97 \pm 0.41$ & $1111.71 \pm 28.37$ \\
\hline M25 & $47.44 \pm 0.62$ & $84.99 \pm 0.22$ & $81.83 \pm 0.34$ & $1159.15 \pm 23.20$ \\
\hline M26 & $6.36 \pm 0.35$ & $32.77 \pm 0.10$ & $48.53 \pm 0.21$ & $36.41 \pm 0.70$ \\
\hline M27 & $15.38 \pm 0.79$ & $78.45 \pm 0.97$ & $66.59 \pm 0.54$ & $113.49 \pm 1.12$ \\
\hline M28 & $8.98 \pm 0.50$ & $24.10 \pm 0.34$ & $60.03 \pm 0.20$ & $196.00 \pm 0.98$ \\
\hline M29 & $17.62 \pm 0.36$ & $29.73 \pm 0.76$ & $77.35 \pm 0.45$ & $265.23 \pm 1.33$ \\
\hline M30 & $20.14 \pm 0.91$ & $78.48 \pm 1.38$ & $81.25 \pm 1.03$ & $145.68 \pm 4.49$ \\
\hline M31 & $47.95 \pm 0.62$ & $100.76 \pm 0.94$ & $110.55 \pm 2.21$ & $1264.94 \pm 16.74$ \\
\hline M32 & $134.17 \pm 0.44$ & $167.50 \pm 1.94$ & $200.29 \pm 4.34$ & $867.12 \pm 20.05$ \\
\hline M33 & $11.89 \pm 0.28$ & $19.40 \pm 0.23$ & $59.92 \pm 0.81$ & $172.56 \pm 3.87$ \\
\hline J01 & $2.80 \pm 0.11$ & $14.82 \pm 0.30$ & $75.60 \pm 1.67$ & $40.82 \pm 3.56$ \\
\hline J02 & $10.70 \pm 0.27$ & $34.44 \pm 0.32$ & $103.39 \pm 1.90$ & $130.67 \pm 7.22$ \\
\hline J03 & $4.72 \pm 0.11$ & $15.42 \pm 0.58$ & $67.33 \pm 0.89$ & $90.02 \pm 2.58$ \\
\hline J04 & $3.30 \pm 0.07$ & $13.43 \pm 0.20$ & $52.40 \pm 2.47$ & $52.23 \pm 1.35$ \\
\hline J05 & $2.79 \pm 0.10$ & $13.78 \pm 0.21$ & $53.72 \pm 0.96$ & $30.75 \pm 0.72$ \\
\hline J06 & $18.41 \pm 0.32$ & $79.75 \pm 1.62$ & $79.59 \pm 5.22$ & $233.35 \pm 8.57$ \\
\hline J07 & $34.50 \pm 0.28$ & $84.60 \pm 4.51$ & $104.13 \pm 2.22$ & $412.22 \pm 8.22$ \\
\hline J08 & $30.34 \pm 0.54$ & $81.59 \pm 5.03$ & $78.20 \pm 2.69$ & $366.76 \pm 6.00$ \\
\hline J09 & $11.93 \pm 0.15$ & $64.74 \pm 2.11$ & $67.56 \pm 2.84$ & $121.43 \pm 6.42$ \\
\hline J10 & $16.55 \pm 0.79$ & $20.17 \pm 0.72$ & $65.93 \pm 4.12$ & $109.34 \pm 6.00$ \\
\hline J11 & $19.94 \pm 0.57$ & $30.32 \pm 0.46$ & $60.58 \pm 3.50$ & $136.72 \pm 9.02$ \\
\hline J12 & $24.10 \pm 0.46$ & $56.29 \pm 1.26$ & $64.93 \pm 1.93$ & $202.14 \pm 4.04$ \\
\hline J13 & $12.28 \pm 0.28$ & $49.24 \pm 0.13$ & $67.56 \pm 1.32$ & $266.34 \pm 3.39$ \\
\hline
\end{tabular}

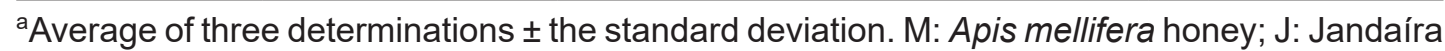
(Melipona interrupta) honey. 
Table IV. Average Cd, Co, $\mathrm{Ni}, \mathrm{Fe}, \mathrm{Mn}, \mathrm{Cr}, \mathrm{Al}$, and $\mathrm{Ba}$ concentrations in the honey samples $\left(\mu \mathrm{g} \mathrm{g}^{-1}\right)$ and their standard deviations ${ }^{a}$

\begin{tabular}{|c|c|c|c|c|c|c|c|c|}
\hline ID & Cd & Co & $\mathbf{N i}$ & $\mathrm{Fe}$ & Mn & $\mathrm{Cr}$ & Al & $\mathrm{Ba}$ \\
\hline M01 & $<$ LOD & $0.13 \pm 0.04$ & $0.07 \pm 0.01$ & $<$ LOD & $1.65 \pm 0.07$ & $<$ LOD & $<L O D$ & $<$ LOD \\
\hline M02 & $0.016 \pm 0.003$ & $0.26 \pm 0.03$ & $<$ LOD & $<$ LOD & $1.52 \pm 0.02$ & $<$ LOD & $<$ LOD & $<$ LOD \\
\hline M03 & $<$ LOD & $0.20 \pm 0.02$ & $0.20 \pm 0.01$ & $<$ LOD & $0.65 \pm 0.01$ & $<$ LOD & $<$ LOD & $<$ LOD \\
\hline M04 & $0.006 \pm 0.001$ & $0.28 \pm 0.02$ & $<$ LOD & $<$ LOD & $1.50 \pm 0.04$ & $<$ LOD & $<$ LOD & $<$ LOD \\
\hline M05 & $0.031 \pm 0.002$ & $0.17 \pm 0.02$ & $0.02 \pm 0.00$ & $<$ LOD & $1.87 \pm 0.04$ & $<$ LOD & $<$ LOD & $<$ LOD \\
\hline M06 & $<$ LOD & $0.06 \pm 0.02$ & $0.03 \pm 0.01$ & $0.42 \pm 0.02$ & $0.99 \pm 0.05$ & $<$ LOD & $0.61 \pm 0.01$ & $<$ LOD \\
\hline M07 & $0.018 \pm 0.004$ & $0.37 \pm 0.02$ & $0.26 \pm 0.02$ & $1.18 \pm 0.09$ & $2.35 \pm 0.09$ & $<$ LOD & $1.84 \pm 0.02$ & $0.14 \pm 0.01$ \\
\hline M08 & $0.011 \pm 0.002$ & $0.20 \pm 0.03$ & $0.05 \pm 0.01$ & $0.40 \pm 0.03$ & $1.33 \pm 0.05$ & $<$ LOD & $0.43 \pm 0.02$ & $<$ LOD \\
\hline M09 & $<$ LOD & $0.25 \pm 0.02$ & $0.02 \pm 0.00$ & $<$ LOD & $1.71 \pm 0.07$ & $0.03 \pm 0.00$ & $<$ LOD & $<$ LOD \\
\hline M10 & $0.031 \pm 0.005$ & $0.37 \pm 0.02$ & $0.10 \pm 0.01$ & $<$ LOD & $1.36 \pm 0.03$ & $0.01 \pm 0.00$ & $<$ LOD & $<$ LOD \\
\hline M11 & $0.063 \pm 0.002$ & $0.21 \pm 0.03$ & $0.20 \pm 0.01$ & $0.13 \pm 0.09$ & $0.50 \pm 0.02$ & $<$ LOD & $0.37 \pm 0.01$ & $<$ LOD \\
\hline M12 & $0.008 \pm 0.002$ & $0.14 \pm 0.02$ & $<$ LOD & $<$ LOD & $0.61 \pm 0.04$ & $<$ LOD & $<$ LOD & $<$ LOD \\
\hline M13 & $<$ LOD & $0.34 \pm 0.08$ & $0.23 \pm 0.03$ & $0.12 \pm 0.02$ & $1.08 \pm 0.04$ & $<$ LOD & $<$ LOD & $<$ LOD \\
\hline M14 & $0.012 \pm 0.003$ & $<$ LOD & $<$ LOD & $0.20 \pm 0.02$ & $0.66 \pm 0.05$ & $<$ LOD & $0.44 \pm 0.01$ & $<$ LOD \\
\hline M15 & $0.045 \pm 0.005$ & $0.06 \pm 0.01$ & $<$ LOD & $0.74 \pm 0.07$ & $1.39 \pm 0.07$ & $<$ LOD & $0.54 \pm 0.01$ & $<$ LOD \\
\hline M16 & $0.057 \pm 0.008$ & $0.07 \pm 0.02$ & $0.05 \pm 0.01$ & $<L O D$ & $0.75 \pm 0.02$ & $0.05 \pm 0.01$ & $0.13 \pm 0.01$ & $<L O D$ \\
\hline M17 & $0.005 \pm 0.001$ & $0.13 \pm 0.02$ & $<$ LOD & $<$ LOD & $3.48 \pm 0.26$ & $<$ LOD & $0.14 \pm 0.01$ & $0.41 \pm 0.03$ \\
\hline M18 & $0.020 \pm 0.003$ & $0.4 \pm 0.04$ & $0.11 \pm 0.01$ & $<$ LOD & $0.43 \pm 0.03$ & $<$ LOD & $1.49 \pm 0.05$ & $<$ LOD \\
\hline M19 & $<$ LOD & $0.31 \pm 0.03$ & $0.20 \pm 0.01$ & $<$ LOD & $0.47 \pm 0.01$ & $0.01 \pm 0.00$ & $0.14 \pm 0.01$ & $0.15 \pm 0.008$ \\
\hline M20 & $<$ LOD & $0.10 \pm 0.02$ & $<$ LOD & $<$ LOD & $0.21 \pm 0.02$ & $<$ LOD & $0.14 \pm 0.02$ & $<$ LOD \\
\hline M21 & $<$ LOD & $0.03 \pm 0.01$ & $0.22 \pm 0.02$ & $3.48 \pm 0.10$ & $0.38 \pm 0.02$ & $<$ LOD & $1.06 \pm 0.05$ & $<$ LOD \\
\hline M22 & $0.002 \pm 0.001$ & $0.36 \pm 0.02$ & $<$ LOD & $3.28 \pm 0.09$ & $0.25 \pm 0.01$ & $<$ LOD & $0.97 \pm 0.01$ & $<$ LOD \\
\hline M23 & $0.003 \pm 0.001$ & $0.02 \pm 0.01$ & $0.16 \pm 0.02$ & $2.66 \pm 0.06$ & $0.61 \pm 0.01$ & $<$ LOD & $3.57 \pm 0.03$ & $<$ LOD \\
\hline M24 & $<$ LOD & $0.05 \pm 0.01$ & $<$ LOD & $2.52 \pm 0.05$ & $0.92 \pm 0.02$ & $<$ LOD & $4.14 \pm 0.02$ & $<$ LOD \\
\hline M25 & $<$ LOD & $<$ LOD & $<$ LOD & $2.04 \pm 0.17$ & $1.73 \pm 0.04$ & $<$ LOD & $4.52 \pm 0.02$ & $0.02 \pm 0.01$ \\
\hline M26 & $<$ LOD & $<$ LOD & $<$ LOD & $<$ LOD & $<$ LOD & $<$ LOD & $3.60 \pm 0.04$ & $<$ LOD \\
\hline M27 & $<$ LOD & $<$ LOD & $<$ LOD & $<$ LOD & $0.14 \pm 0.01$ & $<L O D$ & $2.52 \pm 0.02$ & $<$ LOD \\
\hline M28 & $<$ LOD & $<$ LOD & $<$ LOD & $0.46 \pm 0.04$ & $0.17 \pm 0.01$ & $<$ LOD & $2.10 \pm 0.03$ & $<$ LOD \\
\hline M29 & $<$ LOD & $<$ LOD & $<$ LOD & $<$ LOD & $0.32 \pm 0.01$ & $<$ LOD & $1.64 \pm 0.03$ & $<$ LOD \\
\hline M30 & $0.027 \pm 0.003$ & $0.07 \pm 0.01$ & $0.10 \pm 0.01$ & $56.35 \pm 0.13$ & $0.38 \pm 0.03$ & $0.01 \pm 0.00$ & $<$ LOD & $0.003 \pm 0.001$ \\
\hline M31 & $0.021 \pm 0.004$ & $0.03 \pm 0.01$ & $0.32 \pm 0.03$ & $1.00 \pm 0.07$ & $3.43 \pm 0.14$ & $<$ LOD & $1.26 \pm 0.01$ & $<$ LOD \\
\hline M32 & $0.010 \pm 0.001$ & $0.08 \pm 0.01$ & $0.05 \pm 0.00$ & $1.53 \pm 0.09$ & $1.77 \pm 0.03$ & $<$ LOD & $4.49 \pm 0.05$ & $1.26 \pm 0.11$ \\
\hline M33 & $0.007 \pm 0.002$ & $0.24 \pm 0.01$ & $0.04 \pm 0.01$ & $<$ LOD & $0.37 \pm 0.04$ & $<$ LOD & $0.01 \pm 0.00$ & $0.15 \pm 0.02$ \\
\hline J01 & $<$ LOD & $<$ LOD & $<$ LOD & $<$ LOD & $<$ LOD & $<$ LOD & $2.19 \pm 0.04$ & $<$ LOD \\
\hline J02 & $<$ LOD & $<$ LOD & $<$ LOD & $<$ LOD & $0.23 \pm 0.02$ & $<$ LOD & $3.11 \pm 0.05$ & $0.09 \pm 0.02$ \\
\hline J03 & $<$ LOD & $<$ LOD & $<$ LOD & $<$ LOD & $<$ LOD & $<$ LOD & $1.66 \pm 0.03$ & $<$ LOD \\
\hline
\end{tabular}


Table IV. Average Cd, Co, Ni, Fe, Mn, Cr, Al, and Ba concentrations in the honey samples ( $\left.\mu \mathrm{g} \mathrm{g}^{-1}\right)$ and their standard deviations ${ }^{a}$ (Cont)

\begin{tabular}{lcccccccc}
\hline ID & Cd & Co & Ni & Fe & Mn & Cr & Al & Ba \\
\hline J04 & $<$ LOD & $<$ LOD & $<$ LOD & $<$ LOD & $<$ LOD & $<$ LOD & $1.40 \pm 0.04$ & $<$ LOD \\
J05 & $<$ LOD & $<$ LOD & $<$ LOD & $<$ LOD & $<$ LOD & $<$ LOD & $1.62 \pm 0.02$ & $<$ LOD \\
J06 & $<$ LOD & $<$ LOD & $<$ LOD & $<$ LOD & $0.68 \pm 0.05$ & $<$ LOD & $9.13 \pm 0.07$ & $<$ LOD \\
J07 & $<$ LOD & $0.10 \pm 0.01$ & $<$ LOD & $<$ LOD & $0.59 \pm 0.03$ & $<$ LOD & $2.88 \pm 0.04$ & $<$ LOD \\
J08 & $<$ LOD & $<$ LOD & $<$ LOD & $<$ LOD & $0.49 \pm 0.03$ & $<$ LOD & $2.98 \pm 0.03$ & $0.05 \pm 0.01$ \\
J09 & $<$ LOD & $<$ LOD & $<$ LOD & $<$ LOD & $0.14 \pm 0.03$ & $<$ LOD & $2.63 \pm 0.02$ & $<$ LOD \\
J10 & $0.044 \pm 0.005$ & $0.02 \pm 0.01$ & $0.31 \pm 0.02$ & $<$ LOD & $0.13 \pm 0.01$ & $0.02 \pm 0.00$ & $0.33 \pm 0.01$ & $<$ LOD \\
J11 & $0.017 \pm 0.002$ & $0.27 \pm 0.02$ & $0.21 \pm 0.01$ & $0.52 \pm 0.06$ & $0.34 \pm 0.03$ & $<$ LOD & $0.36 \pm 0.01$ & $<$ LOD \\
J12 & $0.015 \pm 0.004$ & $0.01 \pm 0.00$ & $<$ LOD & $7.59 \pm 0.12$ & $0.78 \pm 0.02$ & $<$ LOD & $3.00 \pm 0.03$ & $<$ LOD \\
J13 & $<$ LOD & $0.27 \pm 0.02$ & $<$ LOD & $0.72 \pm 0.03$ & $0.57 \pm 0.02$ & $<$ LOD & $0.76 \pm 0.01$ & $1.38 \pm 0.23$ \\
\hline
\end{tabular}

${ }^{a}$ Average of three determinations \pm standard deviation. ID Sample; M: Apis mellifera honey; J: Jandaíra honey. LOD: limit of detection.

There were also a few potentially toxic trace elements, such as $\mathrm{Cd}, \mathrm{Co}, \mathrm{Ni}, \mathrm{Fe}, \mathrm{Mn}$, and $\mathrm{Al}$, detected in most of the Apis honey samples and some of the Melipona interrupta samples (Table IV). However, there was generally less than $1.00 \mathrm{\mu g} \mathrm{g}^{-1}$ of these elements present, except for Fe, $\mathrm{Mn}$, and $\mathrm{Al}$ in some samples (Table IV).

The correlations between these metals ranged from -0.114 to 0.848 for $\mathrm{Ca}$, which had a negative correlation with $\mathrm{Cr}$ content $\left(\mathrm{r}^{2}=1.3 \%\right)$ and a strong positive correlation with $\mathrm{Mg}$ content $\left(\mathrm{r}^{2}=72.0 \%\right)$ (Table V). The correlations between $\mathrm{Cr}$ and $\mathrm{Na}$, and $\mathrm{K}$ contents whereas $0.749\left(\mathrm{r}^{2}=56.1 \%\right)$ and with $\mathrm{K}$ was $0.772\left(r^{2}=59.6 \%\right)$, this perhaps shows that the composition of these elements at the sampling honeys, increase when the each one increases, therefore in all samples the calcium content was directly influenced by $\mathrm{Na}, \mathrm{Mg}$ and $\mathrm{K}$ contents representing the major total variation of data. The Mn contents show the major value of correlation between a trace metal $(0.605)\left(r^{2}=36.6 \%\right)$ (Table V).

The $\mathrm{Na}$ correlation coefficients ranged from -0.057 to 0.749 , being -0.057 with Co contents and 0.749 with $\mathrm{Ca}$ contents. The $\mathrm{Na}$ had a moderate correlation with $\mathrm{Mn}(0.488)$ among the trace metals, as the $\mathrm{Ca}$ contents. The others macro elements had the ranges of their correlation coefficients: -0.083 with $\mathrm{Fe}$ and 0.772 with $\mathrm{Ca}$ (for $\mathrm{K}$ contents); and -0.019 with $\mathrm{Fe}$ and 0.848 with $\mathrm{Ca}$ (for $\mathrm{Mg}$ contents).

The Co contents showed a weak positive correlation with Ni contents $(0.300)\left(r^{2}=9.0 \%\right)$, however, it presented moderate negative correlation with Al content $(-0.507)\left(r^{2}=25.0 \%\right)$ (Table V). All others elements showed low correlations with others elements with the remark for the coefficients between $\mathrm{Cr}$ and $\mathrm{Cd}(0.634)$ that could represent a risk for the human health. If the continuous monitoring under the same sampling and analysis conditions shows that $\mathrm{Cr}$ or $\mathrm{Cd}$ continue to be present at high levels, then it may indicate that an increase in $\mathrm{Cr}$ leads to an increase in $\mathrm{Cd}$ and vice versa.

The levels of $\mathrm{Cu}, \mathrm{Pb}$ and $\mathrm{Zn}$ in the honey samples were below the limit of detection. Cadmium was detected in $57.60 \%$ and $23.07 \%$ of Apis and Melipona honey samples, respectively, with concentrations ranging from $0.02(\mathrm{M} 22)$ to $0.063(\mathrm{M} 11) \mathrm{\mu g} \mathrm{g}^{-1}$. In addition, Ni and Co were detected in more than half of the Apis mellifera honey, with concentrations ranging from $0.025 \mu^{-1}$ g (M09) to $0.325 \mu g^{-1}$ (M31) $\mu \mathrm{g} \mathrm{g}^{-1}$, and from $0.016 \mu \mathrm{g} \mathrm{g}^{-1}$ (M23) to $0.373 \mu \mathrm{g} \mathrm{g}^{-1}$ (M10), respectively (Table IV). 
Table V. Pearson's coefficients for macro and trace elements

\begin{tabular}{|c|c|c|c|c|c|c|c|c|c|c|c|}
\hline & Al & $\mathrm{Ba}$ & $\mathrm{Ca}$ & Cd & Co & $\mathrm{Cr}$ & $\mathrm{Fe}$ & $\mathrm{K}$ & $\mathrm{Mg}$ & Mn & $\mathrm{Na}$ \\
\hline $\mathrm{Ba}$ & 0.098 & & & & & & & & & & \\
\hline $\mathrm{Ca}$ & 0.288 & 0.327 & & & & & & & & & \\
\hline $\mathrm{Cd}$ & -0.322 & -0.095 & 0.089 & & & & & & & & \\
\hline Co & -0.507 & 0.147 & -0.007 & 0.146 & & & & & & & \\
\hline $\mathrm{Cr}$ & $-0,229$ & -0.073 & -0.114 & 0.634 & 0.120 & & & & & & \\
\hline $\mathrm{Fe}$ & -0.081 & -0.034 & 0.120 & 0.149 & -0.067 & 0.010 & & & & & \\
\hline K & 0.056 & 0.064 & 0.772 & 0.162 & 0.119 & -0.020 & -0.083 & & & & \\
\hline Mg & 0.128 & 0.363 & 0.848 & 0.187 & 0.080 & 0.007 & -0.019 & 0.738 & & & \\
\hline Mn & -0.124 & 0.184 & 0.605 & 0.164 & 0.281 & -0.041 & -0.076 & 0.645 & 0.504 & & \\
\hline $\mathrm{Na}$ & 0.227 & 0.513 & 0.749 & 0.112 & -0.057 & -0.014 & 0.034 & 0.458 & 0.734 & 0.488 & \\
\hline $\mathrm{Ni}$ & -0.253 & -0.081 & 0.092 & 0.357 & 0.300 & 0.212 & 0.070 & 0.244 & 0.132 & 0.209 & 0.072 \\
\hline
\end{tabular}

\section{Multivariate analysis}

Figure 2 shows the scores and loadings plots of macro and trace elements determined in honey samples. The explained variance of PC1 and PC2 was $51.8 \%$. The contents of $\mathrm{Fe}$, Co and $\mathrm{K}$ were similar and had the same weight to separation as well as $\mathrm{Na}, \mathrm{Cd}$ and $\mathrm{Mg}$ and $\mathrm{Ca}, \mathrm{Ba}, \mathrm{Al}$ and $\mathrm{Ni}$. The contents of $\mathrm{Mn}$ and $\mathrm{Cr}$ were isolated. The content of $\mathrm{Cr}$ has a diametrical opposition in relationship to Al, $\mathrm{Ba}$ and $\mathrm{Ca}$ contents, this may explain the low and negative correlation between these variables $(-0,229$ (Cr-Al); -0.073 (Cr-Ba); -0.114 (Cr-Ca)).

The Apis melifera samples had the major of metal contents responsible to distinguish different geographical location while the Melipona interrupta had the majority of samples with contents of Fe, $\mathrm{Co}, \mathrm{K}, \mathrm{Cd}, \mathrm{Mg}$ and $\mathrm{Na}$ very different in relationship to Apis mellifera samples. Only two samples from Melipona interrupta had similar contents of $\mathrm{Mn}$ in comparison to samples from other bee species.

Some samples of Apis mellifera had similar characteristics to the others samples (M26, M27, M28 and M29). The majority of Apis samples had contents of $\mathrm{Ni}, \mathrm{Al}, \mathrm{Ba}$ and $\mathrm{Ca}$ similar. Two samples had the same contents of $\mathrm{Cd}$ and $\mathrm{Mg}$. Some samples of Apis and Melipona had similar Cr contents (M23, M25, M06 and M14; M24, J07 and J13, respectively). One sample of Apis (M32) was an outlier.
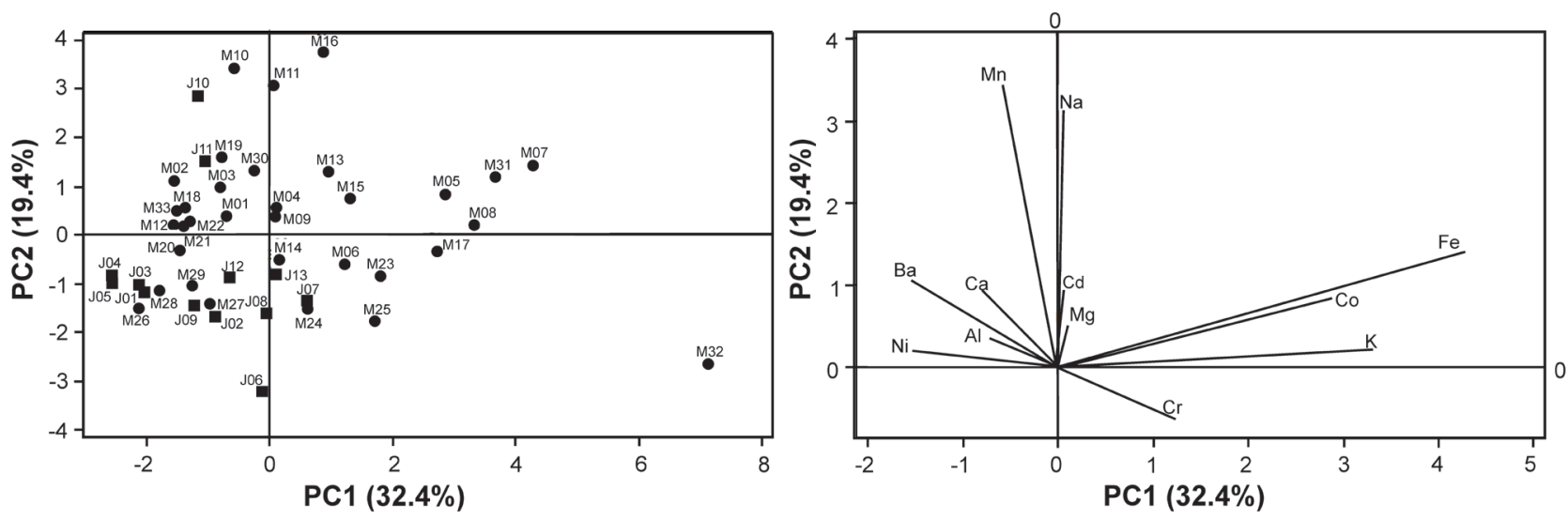

Figure 2. Principal component analysis of the averages of metal concentration in Apis mellifera ( $\mathbf{\square})$ and Melipona interrupta $(\bullet)$ honey. Left: Scores. Right: Loadings. 
The final partition of dendrogram was at $98.1 \%$ similarity (Figure 1). This meant that eight clusters were formed based on the similarity between the samples. The M01, M19, J07, and J08 grouping contained relatively high metal contents, which were similar to the contents in samples M03, M11, and M09 (Figure 3). The major grouping was at 91.8\% similarity (groups II and III, b and c) and this grouping contained 12 sample types. A large number of the " $M$ " samples were around $99.5 \%$ similar; and 14 samples among "J" and "M" sample types had 99.1\% similarity. These sample types were differentiated from the first group where the similarity between the sample types was only $69.9 \%$. The groups included many samples from the different types of bees and therefore formed a heterogeneous cluster.

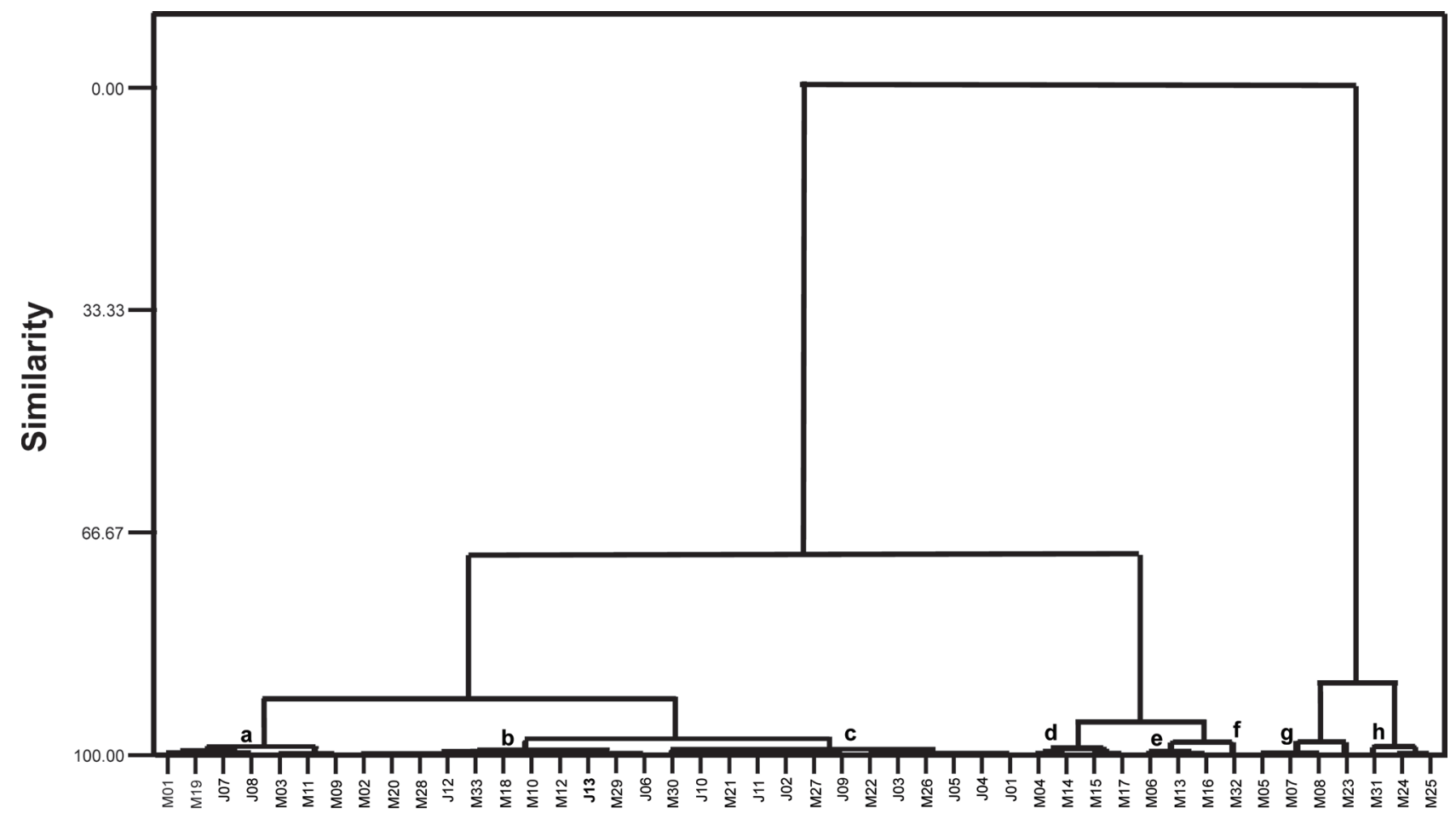

Figure 3. Dendrogram of the macro and trace element concentrations in the honey samples. M: Apis mellifera honey and J: Melipona interrupta honey.

Others sample types grouped into three clusters with $95.2 \%$ similarity, Cluster IV (d) included M04 and $\mathrm{M} 14$, which had similar $\mathrm{K}$ contents, and M15 and M17, which had high $\mathrm{K}$ content similarities with M04 and M14. Cluster V (e) contained sample types M13, M06, and M16 because they had similar Na, $\mathrm{K}$, and Mn contents (Table III). Cluster VI (f) contained just one sample type (M32). This sample was separated from the others because it had high macro-element contents (Table III). Clusters V and VI had $98.1 \%$ similarity. The similarity between these two clusters was lower than between the previous groups and there was no similarity between Clusters $\mathrm{V}$ and $\mathrm{VI}$ and the other groups.

Group VII (g) contained M05, M07, M08, and M23, which all had similar K contents (Table III). The Fe content was higher than in the other clusters, which may explain the reduced similarity between this cluster and the others (Table III). This cluster had $89.8 \%$ similarity with group VIII (h), which contained M31, M24, and M25. The sample types had similar trace element compositions, but M31 had different $\mathrm{Ca}, \mathrm{Na}$, and $\mathrm{K}$ contents. Therefore, these elements were the factor that distinguished M31 from the others samples (Table III). In addition, the "J" samples were not grouped into their own clusters by the analysis. Instead, they were distributed among the "M" samples (Figure 3). 


\section{Discussion}

Since there is no certified and appropriate commercial reference material for honey, the recovery rate was estimated by the use of spiked concentrations of all elements investigated. The percentage of recovery of the analyzed elements was high (ranging from $67 \%$ to $113 \%$ at a spiking concentration of $500 \mu \mathrm{g} \mathrm{g}^{-1}$ ), indicating that the method is suitable.

It is clear that all the honey samples analyzed in this study had total mineral contents that were similar to those found by Silva et al. (2013) in northwestern Pará, Brazil. Silva et al. (2013) also found lower amounts of these minerals in Melipona fasciculata samples compared to Apis mellifera and other species of meliponae in the state of Pará [4].

In general, the macro element levels in the analyzed samples were similar to those found in Brazilian honey [4,19-20], as well as in Spanish multifloral/monofloral honey [1], Portuguese multifloral and monofloral honey [21] and Malaysian honey [22]. The trace elements levels were in agreement with those found in other studies $[4,10]$. The strong correlation between $\mathrm{Ca}$ and $\mathrm{Mg}$ content could be related to plant growth and the close relationship between $\mathrm{Ca}$ and $\mathrm{Mg}$ uptake. Interesting, the relationship between $\mathrm{Ca}$ and $\mathrm{Mn}$ content is reported to be a potential fingerprint for honeys from different regions [23].

The presence of heavy metal in honeys is related to industrial pollution levels near to the apiaries, which means that honey could potentially be used as a biological pollution indicator [24]. The negative correlation between $\mathrm{Co}$ and $\mathrm{Al}$ could be a good potential indicator because when Co increases in the honey Al decreases and vice versa. The presence of these heavy metals may be due to intense agricultural activity close to the nectar collecting regions or to contamination of the groundwater absorbed by trees in the nectar collection area [3]. Cadmium levels were not thought to be high in honey [25]. However, in this study, the positive correlation between these elements was significant and represented $40 \%$ of the determination coefficient. Furthermore, Cd levels were similar to those found in Polish honey from an industrialized region [26] and were higher than those found in French [27] and Brazilian honey [2]. Nevertheless, no honey sample analyzed in this study had Cd concentrations that were higher than the maximum recommended $\left(0.1 \mu \mathrm{g} \mathrm{g}^{-1}\right)$ by international law [28]. Presence of this trace element may indicate anthropogenic activities or the impact of environmental pollution [29-30].

The presence of higher Fe and Al contents in the samples analyzed in this study could be associated with the predominance of latosols and argisols in the study region, which are rich in these metals [31]. Although the $\mathrm{Fe}, \mathrm{Mn}$, and $\mathrm{Al}$ concentrations were higher than the other trace metals [32].

The absence of $\mathrm{Pb}, \mathrm{Cu}$, and $\mathrm{Zn}$ in the analyzed samples corroborated the results obtained by Silva et al. (2013) when they analyzed 27 samples from the North region of Brazil [4]. The presence of Cd, Co, $\mathrm{Fe}, \mathrm{K}, \mathrm{Mg}, \mathrm{Mn}, \mathrm{Pb}$ and $\mathrm{Na}$ in higher concentrations may also be associated with substances provided for the nutrition of bees (e.g. industrial syrups) [6,33]. Most of the metals present in honey come from plants that produce soil and nectar. However, the presence of some metals, mainly $\mathrm{Cd}, \mathrm{Cr}, \mathrm{Cu}, \mathrm{Fe}, \mathrm{Ni}$, $\mathrm{Pb}$ and $\mathrm{Zn}$, may indicate environmental pollution or anthropogenic alteration [6].

All mineral content levels found in the honey samples were lower than the maximum established by Brazilian and international law (Cd and Cr $0.1 \mu \mathrm{g} \mathrm{g}^{-1}, \mathrm{~Pb} 0.30 \mu \mathrm{g} \mathrm{g}^{-1}, \mathrm{Ni}^{2} \mu \mathrm{g} \mathrm{g}^{-1}, \mathrm{Cu} 10 \mu \mathrm{g} \mathrm{g}{ }^{-1}, \mathrm{Zn} 50 \mu \mathrm{g}$ $\left.\mathrm{g}^{-1}\right)$ [28].

The M01, M19, J07, and e J08 grouping may be explained by their similar macro element contents ( $\mathrm{Na}, \mathrm{Ca}, \mathrm{Ba}, \mathrm{Mg}$, and K) (Table III). The differences between M03, M11, and M09 may be due their similar macro and trace element contents (Tables III and IV). These sample types may lead to similar contamination results because their trace element contents were almost the same. The high similarity between the 32 samples in Groups I and II may be due to the high homogeneity between the macro and trace metal contents in each sample type.

The absence of clusters among the Melipona interrupta samples (Figure 2) may indicate that the individual Melipona interrupta sample types were highly heterogeneous, which suggests that floral nectar composition was highly variable and that there was a relationship between the "J" samples and 
the composition of the Apis mellifera samples. The trace-elements in a soil affect honey composition and the macro elements are influenced by the floral origins of the nectar [18]. The high similarity between some "J" and "M" samples may indicate that the soil in the area and the floral nectar collected by the different bee species were similar (Figure 2).

\section{CONCLUSIONS}

The results produced by this study showed that the total mineral contents in the analysed honey were similar to those reported by previous studies in the same region and from other Brazilian regions. The most abundant elements in the honey samples were $\mathrm{K}, \mathrm{Ca}, \mathrm{Mg}$, and $\mathrm{Na}$. The $\mathrm{K}$ contents in the Melipona interrupta honey samples were generally lower than in the Apis mellifera honey. In addition, $\mathrm{Cu}, \mathrm{Pb}$, and $\mathrm{Zn}$ were not detected in any honey sample analysed.

Potentially toxic trace elements, such as, Cd, Co and Ni were detected in most of the Apis mellifera honey samples, and they could be associated with the use of agrochemicals in areas near to the beehives and in the Apis mellifera flightpaths.

The Pearson's coefficients showed that $\mathrm{Mg}, \mathrm{Na}$, and $\mathrm{Mn}$ were highly positively correlated with Ca. In addition, there was a moderate positive correlation between $\mathrm{Ni}$ and $\mathrm{Cd}$ and a moderate negative correlation between Co and Al.

The PCA of the macro and trace elements showed that the mineral content of the samples changes with respect to geographical origin. However, there is no specific separation between honeys of different species.

The HCA of the macro and trace elements showed that the honey sample types could be aligned into eight groups. However, the Melipona interrupta honey samples did not form an isolated group, which may indicate a potentially high variation in floral nectar composition or that the honeys produced by these species may have been altered by the addition of Apis mellifera honey.

Agricultural activities are increasing in the Lower Amazonian region, which means that more research should be conducted periodically to determine the concentrations of potentially toxic heavy metals and to monitor environmental contamination in this region.

\section{Conflict of Interest Statement}

The authors declare no conflict of interest.

\section{Acknowledgements}

This work was supported by Project 88881.159143/2017-01 funded by CAPES (Coordination of Improvement of Higher Education Personnel) and FAPESPA (Amazonia Foundation for Studies and Research in Pará). The authors also thank the two anonymous reviewers for their constructive comments and additions.

Manuscript submitted: Dec. 13, 2018; revised manuscript submitted: June 7, 2019; revised for the $2^{\text {nd }}$ time submitted: July 16, 2019; manuscript accepted: July 19, 2019; published online: Aug. 6, 2019.

\section{REFERENCES}

1. Hernández, O. M.; Fraga, J. M. G.; Jiménez, A. I.; Jiménez, F.; Arias, J. J. Food Chem., 2005, 93, pp 449-458 (DOI: http://dx.doi.org/10.1016/j.foodchem.2004.10.036).

2. Batista, B. L.; Silva, L. R. S.; Rocha, B. A.; Rodrigues, J. L.; Berretta-Silva, A. A.; Bonates, T. O.; Barbosa, F. Food Res. Int., 2012, 49, pp 209-215 (DOI: http://dx.doi.org/10.1016/j. foodres.2012.07.015).

3. Solayman, M.; Islam, M. A.; Paul, S.; Ali, Y.; Khalil, M. I.; Alam, N.; Gan, S. H. Compr. Rev. Food Sci. Food Saf., 2015, 15, pp 219-233 (DOI: http://dx.doi.org/10.1111/1541-4337.12182). 
4. Silva, A. S.; Alves, C. N.; Fernandes, K. G.; Müller, R. C. S. J. Braz. Chem. Soc., 2013, 24, pp 1135-1145 (DOI: http://dx.doi.org/10.5935/0103-5053.20130147).

5. Bogdanov, S.; Haldimann, M.; Luginbühl, W.; Gallmann, P. J. Apic. Res., 2007, 46, pp 269-275 (DOI: http://dx.doi.org/10.1080/00218839.2007.11101407).

6. Pohl, P. TrAC, Trends Anal. Chem., 2009, 28, pp 117-128 (DOI: http://dx.doi.org/10.1016/j. trac.2008.09.015).

7. FAO. 2014. Food and Agriculture Organization of the United Nations. Available from: http://www. fao.org/3/a-i7465e.pdf [Acessed 05 July 2017].

8. Costa, L. S. M.; Albuquerque, M. L. S.; Trugo, L. C.; Quinteiro, L. M. C.; Barth, O. M.; Ribeiro, M.; Maria, C. A. B. Food Chem., 1999, 65, pp 347-352 (DOI: http://dx.doi.org/10.1016/S03088146(98)00230-1).

9. Martini, D. Z.; Moreira, M. A.; Aragão, L. E. O. C.; Formaggio, A. R.; Dalla-Nora, E. L. Land Use Policy, 2015, 49, pp 35-42 (DOI: http://dx.doi.org/10.1016/j.landusepol.2015.07.005).

10. Sodré, G. S.; Marchini, L. C.; Moreti, A. C. C. C.; Otsuk, I. P.; Carvalho, C. A. L. Cienc. Rural, 2007, 37, pp 1139-1144 (DOI: http://dx.doi.org/10.1590/S0103-84782007000400036).

11. Pohl, P.; Sergiel, I.; Stecka, H. Crit. Rev. Anal. Chem., 2009, 39, pp 276-288 (DOI: http://dx.doi. org/10.1080/10408340903001250).

12. Ferreira, S. L. C.; Andrade, J. B.; Korn, M. G. A.; Pereira, M. G.; Lemos, V. A.; Santos, W. N. L.; Silva, E. G. P. J. Hazard. Mater., 2007, 145, pp 358-367 (DOI: http://dx.doi.org/10.1016/j. jhazmat.2007.03.077).

13. Pierini, G. D.; Granero, A. M.; Nezio, M. S.; Centurión, M. E.; Zon, M. A.; Fernández, H. Microchem. J., 2013, 106, pp 102-106 (DOI: http://dx.doi.org/10.1016/j.microc.2012.05.015).

14. Fernández-Torres, R.; Pérez-Bernal, J. L.; Bello-López, M. A.; Callejón-Mochón, M.; JimenézSánchez, J. C.; GuiraúmPérez, A. Talanta, 2005, 65, pp 686-691 (DOI: http://dx.doi.org/10.106/j. talanta.2004.07.030).

15. Corbella, E.; Cozzolino, D. LWT - Food Sci. Technol., 2006, 39, pp 534-539 (DOI: http://dx.doi. org/10.1016/j.Iwt.2005.03.011).

16. Urška, K.; Jasna, B.; Mojca, K.; Marijan, N.; Peter, K.; Nives, O.; Terezija, G. Apiacta, 2009, 44, pp 33-42.

17. Sodré, G. S.; Marchini, L. C.; Moreti, A. C.; Carvalho, C. A. L. Arch. Latinoam. Prod. Anim., 2003, 11, pp 129-137.

18. Jandrić, Z.; Haughey, S. A.; Frew, R. D.; McComb, K.; Galvin-King, P.; Elliott, C. T.; Cannavan, A. Food Chem. 2015, 189, pp 52-59 (DOI: http://dx.doi.org/10.1016/j.foodchem.2014.11.165).

19. Mendes, T. M. F. F.; Baccan, N.; Cadore, S. J. Braz. Chem. Soc., 2006, 17, pp 168-176 (DOI: http://dx.doi.org/10.1590/S0103-50532006000100024).

20. Santos, J. S.; Santos, N. S.; Santos, M. L. P.; Santos, S. N.; Lacerda, J. J. J. J. Braz. Chem. Soc., 2008, 19, pp 502-508 (DOI: http://dx.doi.org/10.1590/S0103-50532008000300018).

21. Silva, L. R.; Videira, R.; Monteiro, A. P.; Valentão, P.; Andrade, P. B. Microchem. J., 2009, 93, pp 73-77 (DOI: http://dx.doi.org/10.1016/j.microc.2009.05.005).

22. Chua, L. S.; Abdul-Rahaman, N. L.; Sarmidi, M. R.; Aziz, R. Food Chem., 2012, 135, pp 880-887 (DOI: http://dx.doi.org/10.1016/j.foodchem.2012.05.106).

23. Golob, T.; Doberšek, U.; Kump, P.; Nečemer, M. Food Chem., 2005, 91, pp 593-600 (DOI: http:// dx.doi.org/10.1016/j.foodchem.2004.04.043).

24. Yücel, Y.; Sultanoglu, P. Food Chem., 2012, 135, pp 170-178 (DOI: http://dx.doi.org/10.1016/j. foodchem.2012.04.061).

25. Akbulut, M.; Özcan, M.; Çoklar, H. Int. J. Food Sci. Nutr., 2009, 60, pp 577-589 (DOI: http://dx.doi. org/10.3109/09637480801892486).

26. Roman, A.; Popiela-Pleban, E.; Migdał, P.; Kruszyński, W. Open Chem., 2016, 14, pp 33-36 (DOI: http://dx.doi.org/10.1515/chem-2016-0007). 
27. Losfeld, G.; Saunier, J. B.; Grison, C. Food Chem., 2014, 146, pp 455-459 (DOI: http://dx.doi. org/10.1016/j.foodchem.2013.08.105).

28. Codex Alimentarius. Revised Codex Standard for Honey, Standards and Standard Methods. Codex Alimentarius Commission FAO/OMS (Vol. 11), 2001.

29. Karabagias, I. K.; Badeka, A.; Kontakos, S.; Karabournioti, S.; Kontominas, M. G. Food Res. Int., 2014, 55, pp 363-372 (DOI: http://dx.doi.org/10.1016/j.foodres.2013.11.032).

30. Moniruzzaman, M.; Chowdhury, M. A. Z.; Rahman, M. A.; Sulaiman, S. A.; Gan, S. H. BioMed Res. Int., 2014, 2014, pp 1-10 (DOI: http://dx.doi.org/10.1155/2014/359890).

31. Santos, H. G.; Jacomine, P. K. T.; Anjos, L. H. C.; Oliveira, V. A.; Coelho, M. R.; Lumbrelas, J. R.; Cunhas, T. J. F. Sistema Brasileiro de Classificação de Solos. EMBRAPA-SPI, Rio de Janeiro, 2006, p 306.

32. Czipa, N.; Alexa, L.; Phillips, C. J. C.; Kovács, B. Eur. Food Res. Technol., 2018, 244, pp 14391445 (DOI: http://dx.doi.org/10.1016/j.foodres.2012.07.015).

33. Rashed, M. N.; Soltan, M. E. J. Food. Compost. Anal., 2004, 17, pp 725-735 (DOI: http://dx.doi. org/10.1016/j.jfca.2003.10.004) 